SMALL INTESTINE

\title{
Changes in the expression of intestinal iron transport and hepatic regulatory molecules explain the enhanced iron absorption associated with pregnancy in the rat
}

\author{
K N Millard, D M Frazer, S J Wilkins, G J Anderson
}

Gut 2004;53:655-660. doi: 10.1136/gut.2003.031153

Background: Iron absorption increases during pregnancy to cater for the increased iron requirements of the growing fetus.

Aims: To investigate the role of the duodenal iron transport molecules and hepatic regulatory molecules in coordinating the changes in iron absorption observed during pregnancy.

Methods: Rats at various days of gestation and 24-48 hours post-partum were examined for hepatic

See end of article for authors' affiliations

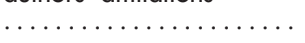

Correspondence to: Dr G J Anderson, Iron Metabolism Laboratory, Queensland Institute of Medical Research, PO Royal Brisbane Hospital, Brisbane, Queensland 4029, Australia; gregA@ qimr.edu.au

Accepted for publication 14 November 2003 expression of hepcidin, transferrin receptors 1 and 2, and HFE (the gene mutated in the most prevalent form of hereditary haemochromatosis), and duodenal expression of divalent metal transporter 1 (DMT1), duodenal cytochrome b (Dcytb), iron regulated mRNA (Ireg1), and hephaestin (Hp) by ribonuclease protection assay, western blotting, and immunohistochemistry.

Results: Decreased hepatic non-haem iron and transferrin saturation and increased expression of transferrin receptor 1 in the liver indicated a progressive reduction in maternal body iron stores during pregnancy. Duodenal expression of the iron transport molecules DMT1, Dcytb, and lregl increased during pregnancy, and this corresponded with a reduction in hepcidin, HFE, and transferrin receptor 2 expression in the liver. Expression of all molecules returned towards control values by 24-48 hours postpartum.

Conclusions: These data indicate that increased expression of key iron transport molecules is responsible for the elevated iron absorption associated with pregnancy, and implicate hepcidin, HFE, and transferrin receptor 2 in determining how the maternal iron homeostatic machinery responds to the increased iron demands accompanying gestation.

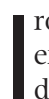
ron requirements increase during pregnancy due to expansion of the maternal erythrocyte mass and high iron demand of the growing fetus. These requirements are met initially through mobilisation of maternal iron stores (principally from the liver) but as iron stores become depleted, intestinal iron absorption increases to maintain an adequate iron supply for both the mother and her offspring. ${ }^{1-3}$ The fetus obtains its iron via the placenta which sequesters transferrin bound iron from the maternal circulation. The rate of materno-fetal transfer increases with the increasing size of the fetus and placenta, and is maximal just prior to parturition. ${ }^{4}$ Iron absorption is also maximal at this time. For example, in humans, there is a ninefold increase in iron absorption between weeks 12 and 36 of gestation ${ }^{6}$ while in rats absorption increases substantially from day 16 through to term at day 21 . $^{1}$ Absorption rapidly returns to control levels post-partum (for example, within 18 hours in rats), ${ }^{7}$ indicating the strength of the absorption stimulus provided by pregnancy.

The ability of the small intestine to respond to the iron demands of pregnancy is indicative of a highly regulated process. Under normal physiological conditions the two main factors that regulate iron absorption are the level of body iron stores and rate of erythropoiesis. ${ }^{8}$ Iron absorption increases when stores are low and decreases when stores are high, so it is not surprising that the depleted body iron levels associated with pregnancy are associated with enhanced iron absorption. However, one study has found that iron loaded rats have increased absorption during pregnancy, in spite of their high iron stores. ${ }^{1}$ This observation suggests that the level of maternal iron stores may not be the sole regulator of iron absorption during pregnancy.
Recent advances in our understanding of the mechanism and regulation of intestinal iron transport have come from the discovery of a number of key molecules involved in the process. These include the iron transporters divalent metal transporter 1 (DMTl) and iron regulated mRNA (Iregl; also known as ferroportin 1), and the enzymes duodenal cytochrome b (Dcytb) and hephaestin (Hp). The brush border transporter DMTl, putative basolateral transporter Ireg l, and the ferrireductase Dcytb are all upregulated under iron deficient conditions ${ }^{9}$ and these changes underlie the increase in iron absorption accompanying iron depletion. Disruption of the ceruloplasmin homologue $\mathrm{Hp}$, a putative iron oxidase, leads to microcytic hypochromic anaemia in mice ${ }^{10}$ but this protein does not appear to be modulated significantly by variations in iron levels. ${ }^{11}$ Several other molecules have been implicated in determining how the body responds to changes in iron requirements, including HFE (the gene mutated in the most prevalent form of hereditary haemochromatosis), transferrin receptor 2 (TfR2), and the antimicrobial peptide hepcidin. These are all highly expressed in the liver and mutations in any of these genes can result in iron overload. ${ }^{12-}$ ${ }^{14}$ Indeed, HFE is the gene most commonly mutated in the prevalent iron loading disorder hereditary haemochromatosis. Hepcidin is secreted into the circulation and has been

Abbreviations: Dcytb, duodenal cytochrome b; DMT1, divalent metal transporter 1; GAPDH, glyceraldehyde 3-phosphate dehydrogenase; $\mathrm{HFE}$, the gene mutated in the most prevalent form of hereditary haemochromatosis; Hp, hephaestin; IRE, iron responsive element; Ireg 1, iron regulated mRNA (also known as ferroportin 1); NP, non-pregnant; $\mathrm{PP}$, post-partum; RPA, ribonuclease protection assay; TfR, transferrin receptor 
proposed to be the messenger between body iron stores and iron absorption by duodenal enterocytes. ${ }^{15}$

There has been no comprehensive analysis of the molecular mechanisms underlying the enhanced iron absorption associated with pregnancy. We thus sought to investigate the role of the duodenal iron transport molecules and hepatic regulatory molecules in coordinating the changes in iron absorption observed as gestation progresses. These studies demonstrated a progressive increase in expression of iron transport molecules in the duodenum during pregnancy that corresponded with decreasing body iron stores and a reduction in liver hepcidin expression. Interestingly, expression of HFE and TfR2 also declined as pregnancy proceeded. After birth, all parameters returned towards control levels within two days.

\section{METHODS}

Animals

Female Sprague-Dawley rats fed a standard rodent pellet diet (370 mg iron $/ \mathrm{kg}$ ) and time mated at 10-12 weeks of age were obtained from the Herston Medical Research Centre, Brisbane, Australia. Rats were sacrificed at 9, 15, 18, and 21 days of gestation, and 24-48 hours post-partum. Those that failed to fall pregnant were used as non-pregnant controls. Rats were anaesthetised (44 mg/kg ketamine and $8 \mathrm{mg} / \mathrm{kg}$ xylazine intraperitoneally) and exsanguinated from the abdominal aorta to provide blood for serum iron determination. Samples of liver and duodenum were removed, and duodenal enterocytes were isolated as described previously. ${ }^{16}$ Tissues and pellets of isolated cells were snap frozen in liquid nitrogen. This study was approved by the Queensland Institute of Medical Research Animal Ethics Committee.

\section{Serum and tissue iron analyses}

Serum transferrin saturation was determined using an iron and iron binding capacity kit (Sigma-Aldrich, Sydney, Australia). Liver tissue was dried overnight at $110^{\circ} \mathrm{C}$, extracted with acid, and non-haem iron content was determined using a colorimetric assay, as described previously. ${ }^{17}$
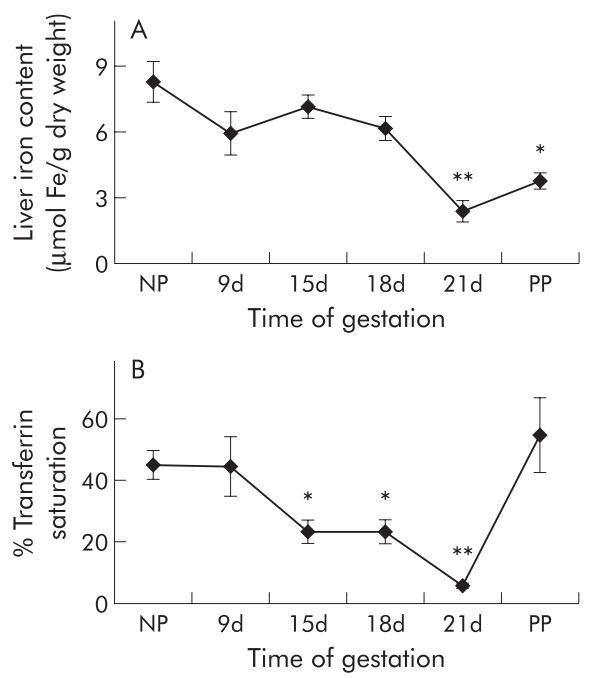

Figure 1 Hepatic non-haem iron and serum transferrin saturation during pregnancy. Rats were studied at various days of gestation and 24-48 hours post-partum (PP) and were compared with non-pregnant rats (NP). Values are reported as $\mu \mathrm{mol}$ non-haem iron/g dry weight liver tissue (A) and percentage saturation of serum transferrin (B). Data are expressed as means (SEM), $n=4$. Significant difference from nonpregnant rats: ${ }^{*} p<0.05,{ }^{* *} p<0.01$.

\section{Ribonuclease protection assays}

Gene expression in liver and enterocytes was analysed by ribonuclease protection assay (RPA) using $5 \mu \mathrm{g}$ of total RNA isolated with TRIzol reagent (Invitrogen, Melbourne, Australia) as per the manufacturer's instructions. Expression of the housekeeping gene glyceraldehyde 3-phosphate dehydrogenase (GAPDH), and the iron metabolism genes $H p, H F E$, hepcidin, Dcytb, Iregl, transferrin receptor 1 (TfRI), TfR2, and DMTI (both the iron responsive element (IRE) containing splice variant and the non-IRE form) were examined using riboprobes corresponding to the complementary DNA sequences of these genes, as previously described. ${ }^{11}$ The riboprobe for TfR2 corresponded to nucleotides 1859 to 2039 of the cDNA sequence listed in GenBank (accession No XM 22022). Band intensities were quantified by densitometry, and iron metabolism gene expression was reported as a proportion of GAPDH gene expression.

\section{Western blot analysis}

Protein was extracted from isolated duodenal enterocytes and DMTl and Iregl protein expression examined by western blotting using polyclonal antibodies raised as previously described. ${ }^{9}{ }^{11} 18$ Band intensities were quantified by densitometry, and expression of DMTl and Iregl were reported as a proportion of actin protein expression to control for any discrepancies in gel loading.

\section{Immunohistochemistry}

Sections cut from duodenal tissue embedded and snap frozen in OCT embedding compound (Sakura Finetek, Torrance, California, USA) were used to localise DMTl and Dcytb proteins by immunofluorescence microscopy, as previously described. ${ }^{9}$

\section{Statistical analysis}

All values are expressed as mean (SEM). Statistical differences between means were calculated using ANOVA and the Student's $t$ test, correcting for differences in sample variance (Microsoft Excel; Microsoft Pty Ltd, North Ryde, Australia).

\section{RESULTS}

As pregnancy progresses and fetal iron demand increases, there is a corresponding drop in maternal iron stores. ${ }^{3}$ In this study, hepatic non-haem iron levels decreased from 8.27 (0.9) $\mu \mathrm{mol}$ iron/g dry weight in non-pregnant control rats to $2.43(0.5) \mu \mathrm{mol}$ iron/g $(\mathrm{p}<0.01)$ on day 21 of gestation (fig 1A). Liver iron content increased slightly after birth to 3.80 (0.4) $\mu \mathrm{mol} \mathrm{Fe/g} \mathrm{but} \mathrm{was} \mathrm{still} \mathrm{well} \mathrm{below} \mathrm{control} \mathrm{levels}$ $(p<0.05)$. Transferrin saturation progressively decreased throughout pregnancy from 45.1 (4.6)\% in non-pregnant animals to $6.5(0.8) \%$ on day $21(\mathrm{p}<0.01)$ but returned to control levels by 48 hours post-partum (fig 1B). Corresponding with this reduction in liver iron concentration and transferrin saturation was an increase in the hepatic expression of $T f R I$ mRNA, as measured by RPA (5.9-fold over non-pregnant level on day 21; $<<0.05$ ) (fig 2). TfRI expression had fallen by $24-48$ hours post-partum but was still 3.5-fold above non-pregnant levels $(p<0.01)$.

As recent studies have suggested that the hepatic molecules hepcidin, HFE, and TfR2 play key roles in the regulation of iron absorption, we investigated their expression during pregnancy. There was a progressive decline in hepcidin expression (fig 2) which reached statistical significance by day 15 and a minimum of $1.9 \%$ of the nonpregnant level $(\mathrm{p}<0.05)$ on day 21 . After birth, hepcidin expression rapidly returned to near control levels. Hepatic expression of both HFE and TfR2 decreased as pregnancy progressed (fig 2). HFE expression followed a similar pattern to hepcidin and was significantly decreased to $18 \%$ 

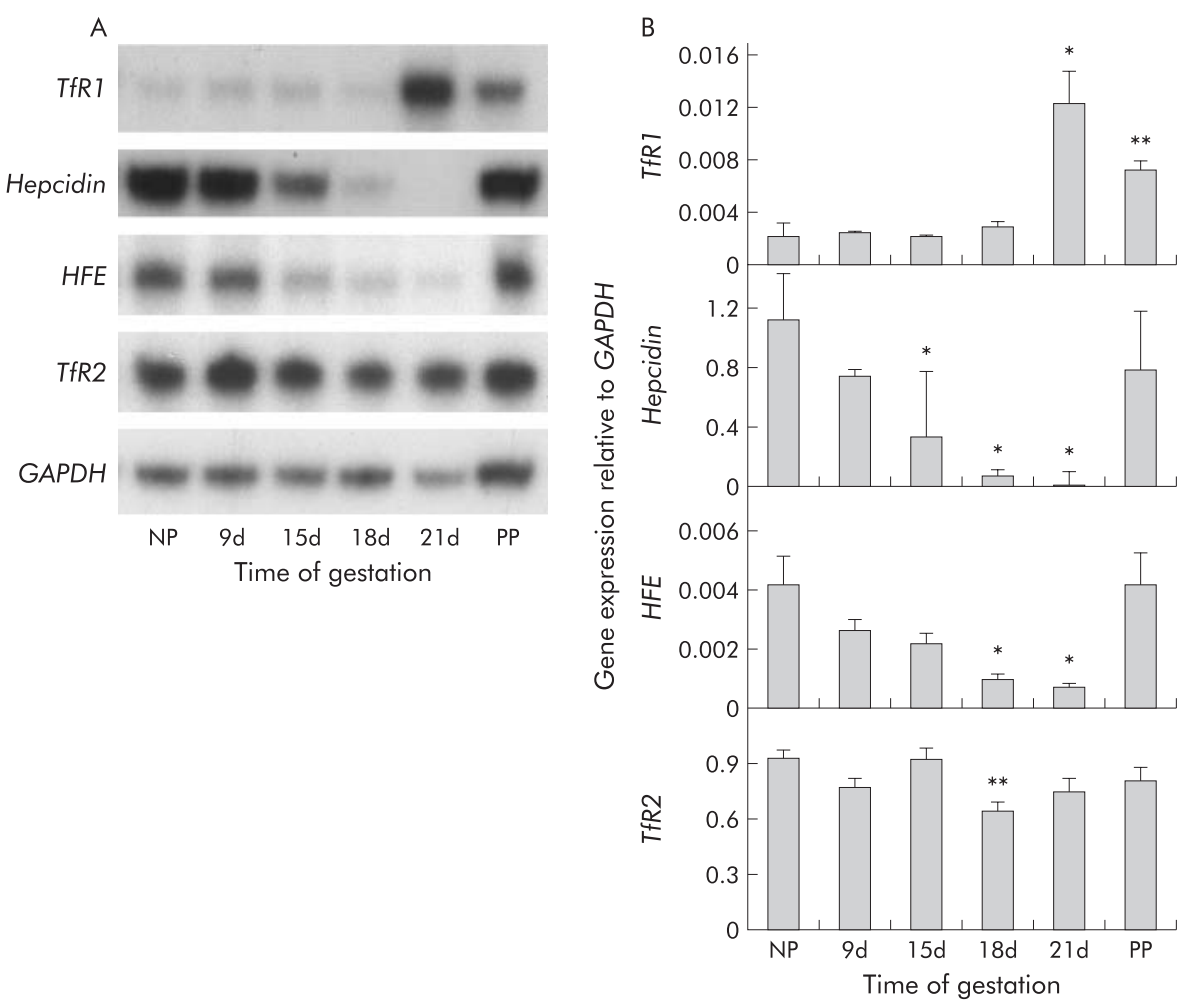

Figure 2 Hepatic expression of transferrin receptor 1 (TfRI), hepcidin, HFE (the gene mutated in the most prevalent form of hereditary haemochromatosis), and TfR2 mRNA. Animals are as described in fig 1. Expression of each gene was measured by ribonuclease protection assay. (A) Representative assays for each gene. (B) Band intensities for each gene were measured by densitometry and graphed as a proportion of glyceraldehyde 3-phosphate dehydrogenase (GAPDH). Data are mean (SEM), $n=4$. Significant difference from non-pregnant rats: * $p<0.05$, ${ }^{* *} \mathrm{p}<0.01$.

of non-pregnancy levels $(\mathrm{p}<0.05$ compared with nonpregnant controls) on day 21 before returning to control levels by $24-48$ hours after birth. The reduction in TfR2 expression was less striking, with a decline to $70 \%$ of the control value on day 18 of pregnancy $(\mathrm{p}<0.01)$.

As an increase in maternal intestinal iron absorption has been well described during pregnancy, ${ }^{16}$ we investigated expression of duodenal iron transport molecules during gestation. Expression of mRNA for the brush border iron transporter DMTl increased as pregnancy progressed (fig 3), peaking at 7.9 times $(\mathrm{p}<0.05)$ and 2.4 times $(\mathrm{p}<0.01)$ nonpregnant levels for the IRE and non-IRE forms, respectively, on gestation day 18, but declining thereafter. Western blot analysis showed DMTl protein levels to be similarly upregulated (8.1-fold over non-pregnant control animals; $\mathrm{p}<0.05$ ) on day 18 at the protein level and reduced to control levels by 24-48 hours post-partum (fig 4). Immunofluorescence showed an increase in DMTl on the brush border on days 18 and 21 compared with both non-pregnant (fig 5) and 24-48 hour post-partum rats. Expression of the brush border ferrireductase Dcytb was also increased in the latter half of pregnancy. At day 15, mRNA expression rose to 2.9 times non-pregnant levels, remained relatively constant until day 21 (3.3-fold increase on day 18 and 3.1-fold increase on day 21 over non-pregnant animals; $\mathrm{p}<0.05$ ) before declining after birth (fig 3). Immunohistochemistry showed little Dcytb staining in non-pregnant animals but distinct brush border staining on days 18 and 21 . This was greatly reduced by $24-48$ hours after birth (fig 5 ).

Iregl mRNA expression showed a small increase in the latter stages of pregnancy but, unlike DMTI and Dcytb, the change was not significant (fig 3). However, the Iregl protein level was increased on day 15 to 1.8 times non-pregnant controls $(p<0.05)$ and this trend continued with 1.6-fold and 1.7 -fold increases over control at days 18 and 21 , respectively $(p=0.06)$. Protein expression returned to control levels after birth (fig 4). Hp mRNA expression was unchanged for the duration of pregnancy and after birth (fig 3).

\section{DISCUSSION}

The growing fetus has a high demand for iron which must be met from maternal sources. As the amount of iron transferred to the fetus throughout the course of pregnancy exceeds the amount of stored maternal iron under normal circumstances, there must be a compensatory increase in maternal iron absorption to provide the extra iron needed. In this study, we have undertaken a preliminary characterisation of this process at the molecular level.

As evidence of increased maternal-fetal transfer and mobilisation of maternal iron, we observed a drop in transferrin saturation and hepatic non-haem iron, and an increase in TfRl expression as pregnancy progressed. This reduction in transferrin saturation and maternal iron stores was accompanied by an increase in the expression of the brush border iron transporter DMTl and the iron reductase Dcytb in the duodenum, with maximum levels being observed in the last few days of gestation. One other group has examined DMTI mRNA expression during pregnancy and they also found highest levels in the latter stages of gestation. ${ }^{19}$ Like DMTl and Dcytb, expression of the basolateral iron exporter Iregl was upregulated in late pregnancy but the increase was only significant at the protein level. Expression of all three molecules fell sharply after birth. There was also an increase in transferrin saturation at this time, thus strengthening the relationship between increased systemic iron demand and maternal 

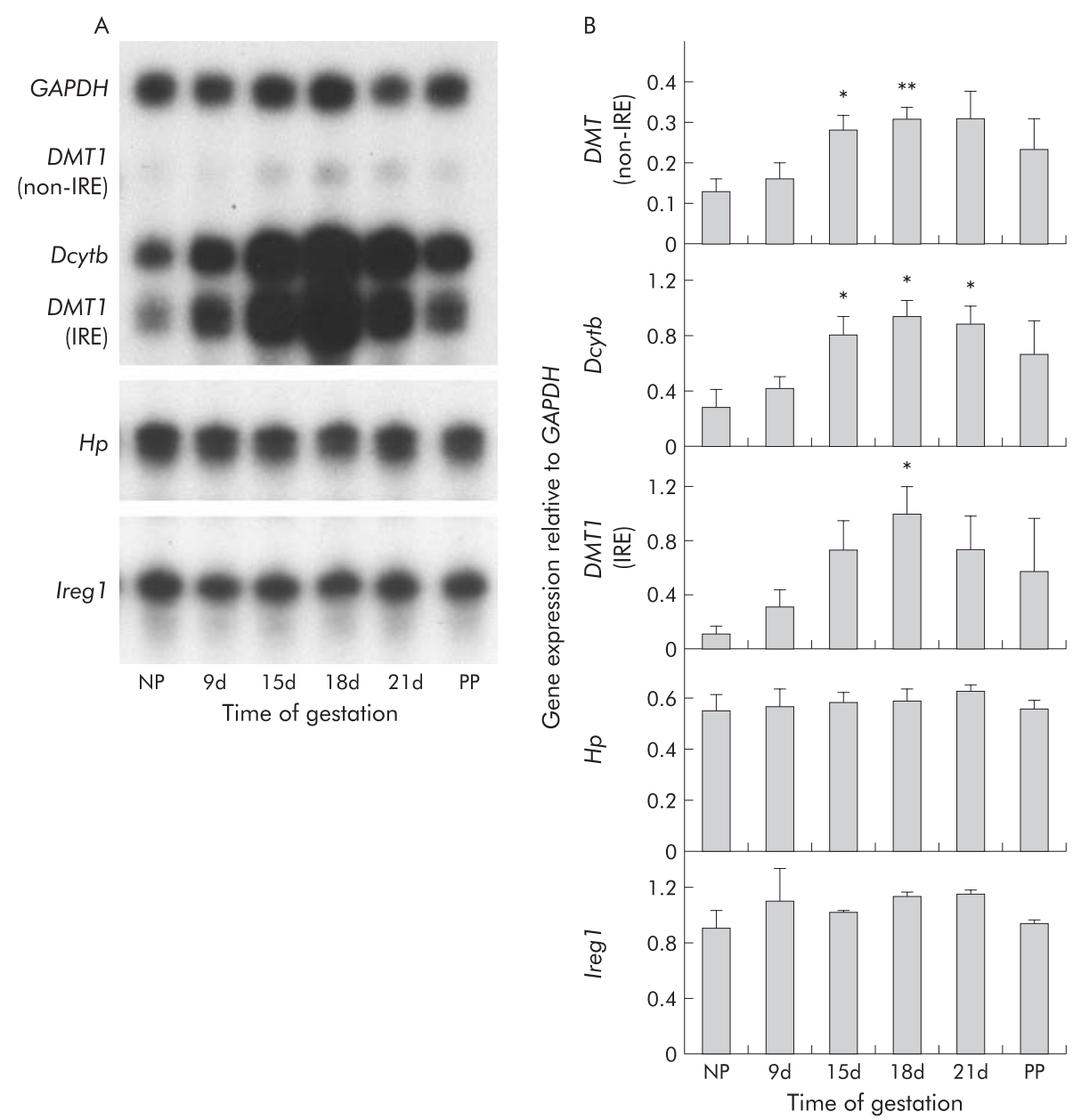

Figure 3 Duodenal expression of divalent metal transporter 1 (DMTI) (iron responsive element (IRE) and non-IRE forms), duodenal cyłochrome b $(D c y+b)$, hephaestin $(H p)$, and iron regulated mRNA (Ireg 1) mRNA. Animals are as described in fig 1. Expression of each gene was measured by ribonuclease protection assay. (A) Representative assays for each gene. (B) Band intensities for each gene were measured by densitometry and graphed as a proportion of glyceraldehyde 3-phosphate dehydrogenase (GAPDH). Data are mean (SEM), $n=4$. Significant difference from nonpregnant rats: ${ }^{*} p<0.05,{ }^{* *} p<0.01$.

transporter expression. There were no significant changes in expression of the iron oxidase $H p$.

The progressive increase in expression of intestinal iron transport molecules as pregnancy advances and the sharp fall in expression after birth are consistent with previous studies showing that iron absorption reaches a maximum in the latter stages of gestation ${ }^{16720}$ and rapidly declines after birth. ${ }^{16}$ These studies have used a variety of techniques to demonstrate absorption changes, including ${ }^{59} \mathrm{Fe}$ absorption through duodenal loops (rats), uptake of ${ }^{59} \mathrm{Fe}$ from the diet (rats), and stable isotope studies (humans). The observed regulation is also consistent with numerous previous investigations showing that increased iron demands and depleted iron stores are associated with increased expression of the intestinal iron transport machinery. ${ }^{11}{ }^{21}$ Other studies have highlighted the fact that while many iron transport molecules are regulated transcriptionally, they can also be regulated translationally and at the cellular level by iron dependent changes in subcellular localisation. ${ }^{1822}$ For example, the latter mode of regulation could be important for Ireg 1 and $\mathrm{Hp}$, as recent data have indicated that the basolateral transfer step of iron absorption is likely to play a key role in how the intestine responds to changes in systemic iron demand. ${ }^{23}{ }^{24}$ Indeed, in this study we found little regulation of Iregl at the mRNA level but a significant increase when protein was studied, suggesting that post-transcriptional mechanisms are most important in the regulation of this molecule during pregnancy.

In order to investigate how the body signals the small intestine to absorb more iron during pregnancy, we investigated expression of the liver derived regulatory peptide hepcidin. Hepcidin expression decreased progressively throughout pregnancy, reaching its lowest level just before parturition, then returned to near normal levels soon after birth. The inverse relationship between expression of hepcidin in the liver and iron transport proteins in the duodenum observed in this study is consistent with a growing body of evidence suggesting that hepcidin acts as a repressor of intestinal iron absorption. Mice and humans with abrogated hepcidin expression develop iron overload ${ }^{1425}$ whereas mice overexpressing the gene develop iron deficiency anaemia. ${ }^{26}$ Similarly, experimentally induced iron overload results in increased hepcidin expression and reduced gut transporter expression while iron deficiency has the opposite effect. ${ }^{11}{ }^{27}$ Our data suggest that hepcidin is a key messenger controlling intestinal iron absorption during pregnancy.

Factors leading to alterations in expression of hepcidin are poorly defined but recent evidence suggests that HFE and TfR2 are potential regulatory molecules. Mutations in either of these genes lead to iron overload subsequent to increased iron absorption. ${ }^{12}{ }^{13}$ Furthermore, hepcidin expression is greatly diminished in HFE associated haemochromatosis, 

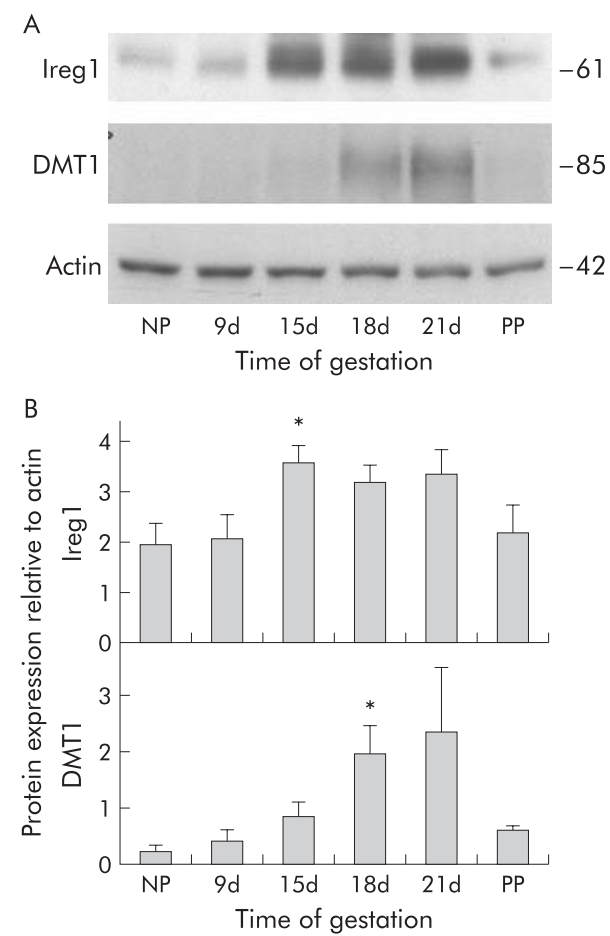

Figure 4 Expression of divalent metal transporter 1 (DMT1) and iron regulated mRNA (Ireg1) protein in the duodenum. Animals are as described in fig 1. The level of each protein was determined by western blotting. (A) Representative western blots for each protein. (B) Band intensities for each protein were measured by densitometry and graphed as a proportion of actin. Data are mean (SEM), $n=4$. Significant difference from non-pregnant rats: ${ }^{*} \mathrm{p}<0.05$.

despite elevated iron stores, ${ }^{28}$ and the same response would be predicted in TfR 2 associated iron overload as patients with HFE, hepcidin, and TfR2 mutations all have very similar phenotypes. Thus HFE (and possibly TfR2) appear to be upstream regulators of hepcidin expression and are required for hepcidin to respond appropriately to changes in body iron levels. Consequently, it was of some interest that we observed a decrease in expression of $H F E$, and to a lesser extent TfR2, mRNA levels during pregnancy. We recently proposed a mechanism by which HFE and TfR2 could respond to variations in transferrin saturation and subsequently influence hepcidin expression. ${ }^{15}$ As maternal iron levels and transferrin saturation are reduced during pregnancy, this is a potential mechanism for influencing hepcidin levels. However, other factors altering HFE and/or TfR2 expression could have a similar effect. In this regard it is notable that Batey and Gallagher ${ }^{1}$ found that iron absorption was still elevated in pregnant rats maintained on an iron loaded diet, albeit not to the same levels as found in animals on a control diet. This suggests that both iron dependent and iron independent factors are involved in determining the absorption response during pregnancy. It is possible that a placenta derived factor could play an important role in this regulation as the placenta is critical for iron delivery to the fetus.

How HFE and TfR2 themselves are regulated is unclear. Previous studies have suggested that expression of HFE is increased in intestinal cells lines with iron loading ${ }^{29}$ and decreased in the intestine in iron deficiency. ${ }^{30}$ However, the range of regulation was much smaller than the range of expression we observed in the liver during pregnancy. It is possible that this is due to tissue specific regulation, and that HFE in the liver is far more responsive to changes in tissue iron content than is HFE in the gut. Alternatively, there may be a pregnancy specific factor involved. In support of this possibility, earlier studies have failed to find evidence of TfR2 regulation in the liver when body iron load is varied, ${ }^{31}$ while we saw a significant decrease in TfR2 expression in late gestation. A candidate pregnancy specific factor is oestrogen. During pregnancy, oestrogen is initially secreted from the corpus luteum and later the placenta. ${ }^{32}$ Oestrogen levels progressively increase during pregnancy, peaking just prior to parturition, the same time that iron absorption is maximal, then dropping dramatically soon after birth (when the placenta is expelled), again similar to absorption. ${ }^{32}{ }^{33}$ In addition, when oestrogen was administered to ovariectomised rats at concentrations similar to those found in late pregnancy, iron absorption was increased. ${ }^{34}$ Whether oestrogen plays a role in the regulation of HFE and/or TfR2 remains to be determined.

In conclusion, the changes in iron absorption that occur during pregnancy are consistent with the increase in duodenal iron transporter expression and the decrease in hepcidin expression observed in this study. Decreased expression of $H F E$ and $T f R 2$ is also likely to be important during gestation and may underlie the hepcidin response. Whether
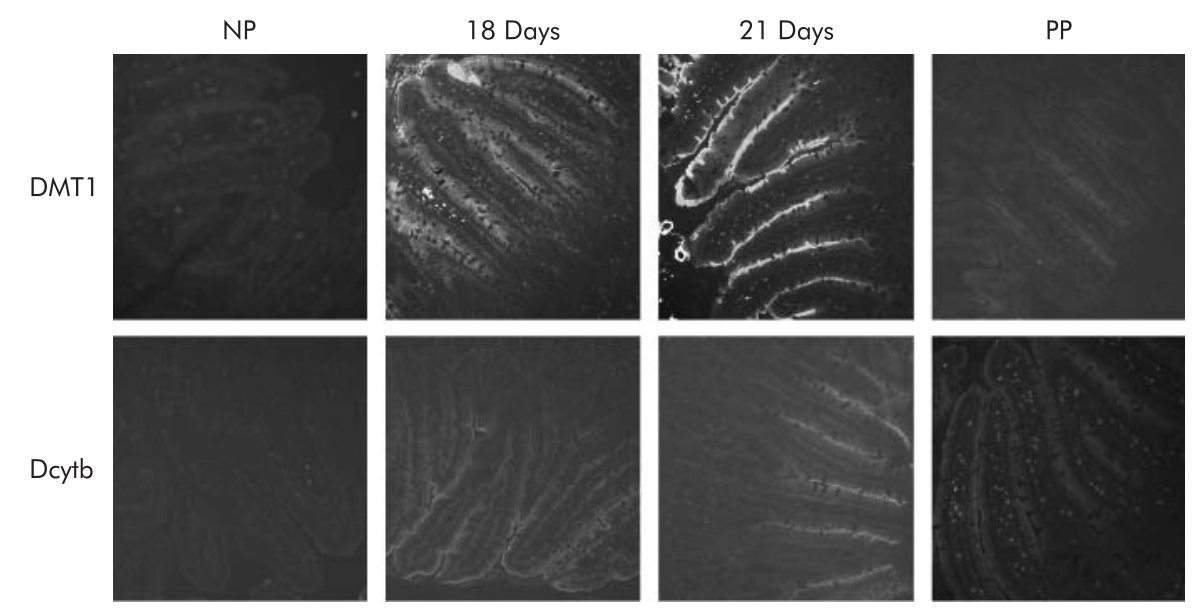

Figure 5 Localisation of duodenal cytochrome b (Dcytb) and divalent metal transporter 1 (DMT1) by immunohistochemistry. Duodenal sections shown are from non-pregnant (NP) rats, pregnant rats at gestation days 18 and 21, and rats $24-48$ hours post-partum (PP). Immunofluorescent staining was carried out using antibodies specific for Dcytb or DMT1. Sections were studied from four animals at each time point and representative sections are shown. Original magnification $100 \times$. 
these molecules respond to maternal iron levels and transferrin saturation, a pregnancy specific factor, or a combination of both, will require further investigation.

\section{ACKNOWLEDGEMENTS}

This work was supported in part by grants from the National Health and Medical Research Council of Australia and the Human Frontier Science Program (grant RGY0328/2001-M).

\section{Authors' affiliations}

K N Millard, D M Frazer, S J Wilkins, G J Anderson, Iron Metabolism Laboratory, The Queensland Institute of Medical Research, PO Royal Brisbane Hospital, Brisbane, Queensland 4029 Australia

\section{REFERENCES}

1 Batey RG, Gallagher ND. Role of the placenta in intestinal absorption of iron in pregnant rats. Gastroenterology 1977:72:255-9.

2 Murray MJ, Stein N. Contribution of maternal rat iron stores to fetal iron in maternal iron deficiency and overload. J Nutr 1971;101:1583-7.

3 van Eijk HG, Kroos MJ, van der HC, et al. Observations on the iron status during pregnancy in rats. Iron transport from mother to fetus. Eur J Obstet Gynecol Reprod Biol 1980;10:389-92.

4 Glasser SR, Wright C, Heyssel RM. Transfer of iron across the placenta and fetal membranes in the rat. Am J Physiol 1968;215:205-10.

5 Kaufman N, Wyllie JC. Maternofoetal iron transfer in the rat. Br J Haematol 1970;19:515-21.

6 Barrett JF, Whittaker PG, Williams JG, et al. Absorption of non-haem iron from food during normal pregnancy. BMJ 1994:309:79-82.

7 Batey RG, Gallagher ND. Study of the subcellular localization of $59 \mathrm{Fe}$ and iron-binding proteins in the duodenal mucosa of pregnant and nonpregnan rats. Gastroenterology 1977;73:267-72.

8 Finch C. Regulators of iron balance in humans. Blood 1994:84:1697-1702.

9 Frazer DM, Wilkins SJ, Becker EM, et al. Hepcidin expression inversely correlates with the expression of duodenal iron transporters and iron absorption in rats. Gastroenterology 2002;123:835-44.

10 Vulpe CD, Kuo YM, Murphy TL, et al. Hephaestin, a ceruloplasmin homologue implicated in intestinal iron transport, is defective in the sla mouse. Nat Genet 1999;21:195-9.

11 Frazer DM, Vulpe CD, McKie AT, et al. Cloning and gastrointestinal expression of rat hephaestin: relationship to other iron transport proteins. Am J Physiol Gastrointest Liver Physiol 2001;281:G931-9.

12 Camaschella C, Roetto A, Cali A, et al. The gene TFR2 is mutated in a new type of haemochromatosis mapping to 7q22. Nat Genet 2000;25:14-15.

13 Feder JN, Gnirke A, Thomas W, et al. A novel MHC class I-like gene is mutated in patients with hereditary haemochromatosis. Nat Genet 1996;13:399-408.

14 Roetto A, Papanikolaou G, Politou M, et al. Mutant antimicrobial peptide hepcidin is associated with severe juvenile hemochromatosis. Nat Genet 2003:33:21-22.
15 Frazer DM, Anderson GJ. The orchestration of body iron intake: how and where do enterocytes receive their cues? Blood Cells Mol Dis 2003;30:288-97.

16 Anderson GJ, Powell LW, Halliday JW. The endocytosis of transferrin by rat intestinal epithelial cells. Gastroenterology 1994;106:414-22

17 Torrance JD, Bothwell TH. A simple technique for measuring storage iron concentrations in formalinised liver samples. S Afr J Med Sci 1968;33:9-1 1 .

18 Trinder D, Oates PS, Thomas C, et al. Localisation of divalent metal transporter 1 (DMT1) to the microvillus membrane of rat duodenal enterocytes in iron deficiency, but to hepatocytes in iron overload. Gut 2000;46:270-6.

19 Leazer TM, Liu Y, Klaassen CD. Cadmium absorption and its relationship to divalent metal transporter-1 in the pregnant rat. Toxicol Appl Pharmacol 2002;185:18-24.

20 Southon S, Wright AJ, Fairweather-Tait SJ. The effect of differences in dietary iron intake on $59 \mathrm{Fe}$ absorption and duodenal morphology in pregnant rats. Br J Nutr 1989;62:707-17.

21 McKie AT, Barrow D, Latunde-Dada GO, et al. An iron-regulated ferric reductase associated with the absorption of dietary iron. Science 2001;291:1755-9.

22 Abboud S, Haile DJ. A novel mammalian iron-regulated protein involved in intracellular iron metabolism. J Biol Chem 2000;275:19906-12.

23 Frazer DM, Wilkins SJ, Becker EM, et al. A rapid decrease in the expression of DMT1 and Dcytb but not Iregl or hephaestin explains the mucosal block phenomenon of iron absorption. Gut 2003;52:340-6.

24 Chen H, Su T, Attieh ZK, et al. Systemic regulation of Hephaestin and Ireg 1 revealed in studies of genetic and nutritional iron deficiency. Blood 2003:102:1893-9.

25 Nicolas G, Bennoun M, Devaux I, et al. Lack of hepcidin gene expression and severe tissue iron overload in upstream stimulatory factor 2 (USF2) knockout mice. Proc Natl Acad Sci U S A 2001;98:8780-5.

26 Nicolas G, Bennoun M, Porteu A, et al. Severe iron deficiency anemia in transgenic mice expressing liver hepcidin. Proc Natl Acad Sci U S A 2002;99:4596-601.

27 Pigeon C, llyin G, Courselaud B, et al. A new mouse liver-specific gene, encoding a protein homologous to human antimicrobial peptide hepcidin, is overexpressed during iron overload. J Biol Chem 2001;276:7811-19.

28 Bridle KR, Frazer DM, Wilkins SJ, et al. Disrupted hepcidin regulation in HFEassociated haemochromatosis and the liver as a regulator of body iron homoeostasis. Lancet 2003;361:669-73.

29 Han O, Fleet JC, Wood RJ. Reciprocal regulation of HFE and Nramp2 gene expression by iron in human intestinal cells. J Nutr 1999;129:98-104.

30 Byrnes V, Ryan E, O'Keane C, et al. Immunohistochemistry of the Hfe protein in patients with hereditary hemochromatosis, iron deficiency anemia, and normal controls. Blood Cells Mol Dis 2000;26:2-8.

31 Fleming RE, Migas MC, Holden CC, et al. Transferrin receptor 2: continued expression in mouse liver in the face of iron overload and in hereditary hemochromatosis. Proc Natl Acad Sci U S A 2000;97:2214-19.

32 Fotherby $F$, The endocrinology of the menstrual cycle, pregnancy, In: Makin HL, e, eds. Biochemistry of steroid hormones. Oxford: Blackwell Scientific Publications, 1975:261-9.

33 de Lauzon S, Uhrich F, Vandel S, et al. Determination of progesterone and of free and conjugated estrogens in pregnant and peudo-pregnant rats. Steroids 1974;24:31-40.

34 Haouari M, Haouari-Oukerro F, Alguemi C, et al. Effects of oestradiol-17 beta on small intestine iron absorption and iron uptake into blood and liver. Horm Metab Res 1994:26:53-4 